\title{
Local Stability and Bifurcation Analysis of A Functionally Graded Material Plate
}

\author{
Xiao-Hua ZHANG ${ }^{1,2, *}$, Yan-Lin HAN ${ }^{1}$ \\ ${ }^{1}$ School of Information Technology, Jiangsu Institute of Commerce, Nanjing 210016, China \\ ${ }^{2}$ Department of Mathematics, Nanjing University of Aeronautic and Astronautic, Nanjing 210016, \\ China \\ *zhangcy11@163.com
}

Keywords: FGM, Stability, Bifurcation, Normal Form.

\begin{abstract}
In this paper, we investigated the local stability of a simply supported functionally graded material (FGM) rectangular plate subjected to the transversal and in-plate excitations in the uniform thermal environment both analytical and numerical approaches. Three kinds of critical points of the bifurcation response equations are considered, which are characterized by a double zero eigenvalues, a simple zero and a pair of pure imaginary eigenvalues as well as two pairs of pure imaginary eigenvalues in nonresonant case, respectively. With the aid of a symbolic computation language Maple and normal form theory, the explicit expressions of critical bifurcation lines are obtained. Finally, the numerical solutions obtained by using fourth-order Runge-Kutta method agree with the analytic predictions.
\end{abstract}

\section{Introduction}

The functionally graded material is one of the extremely excellent material, which is inhomogeneous composite material whose properties varies continuously and smoothly from a ceramic surface to a metallic surface in a specified direction of the structure. The ceramic face protects the metallic surface from corrosion, whereas the metallic part offers strength and stiffness to the structure. Because of the special component, the phenomena of corrosion decrease.

In order to use the functionally graded materials effectively and efficiently, researchers have done various studies on stability, nonlinear vibration, bifurcation and chaos of the functionally graded materials [1-6]. In addition, much work related to complex dynamic bifurcation phenomena exhibited by a nonlinear autonomous system in the vicinity of compound critical points have been discussed by a few researchers in detail. P. Yu and K. Huseyin studied the stability and bifurcation behaviors of the systems with different critical points [7-11]. Later P. Yu et al. [12-17] used the symbolic language Maple based on the method of multiple time scales for computing the corresponding normal forms.

In this paper, we extend the research of paper [18] and consider the stability and local bifurcation behaviors for the FGM rectangular plate in the case of the detuning parameters as perturbation parameters. This paper is organized as follows: In Sect.2, the averaged equations of transverse motion of the FGM plate are given in terms of a set of differential equations and the stability conditions of initial equilibrium solution are obtained explicitly. The detailed stability and bifurcation analysis of the system in the vicinity of the three types of critical points are presented In Sect.3, which is followed in Sect. 4 by a short summary.

\section{Formulation of the problem}

In paper [18], the stability and local bifurcations of the functionally graded material (FGM) rectangular plate on the condition of damping parameters as perturbation parameters have been studied. The figure is shown in Figure 1. The edge width, length and thickness of the FGM rectangular plate in the $x, y$ and $z$ directions are $a, b$ and $h$ respectively. Especially the width-to-length ratio of the FGM rectangular 
plate is $a / b=1$. The transversal excitation subject to the FGM plate is of the form $F(x, y) \cos \Omega_{1} t$ and the in-plate excitation along $x$ direction at $x=0$ and $x=a$ is represented by $p=p_{0}-p_{1} \cos \Omega_{2} t$. Here, $\Omega_{1}$ and $\Omega_{2}$ are the frequencies of the transversal excitation and in-plane excitation, respectively.

In paper [18], the nonlinear governing differential equations of transverse motion of the FGM rectangular plate for the dimensionless are obtained as follows,

$$
\begin{array}{r}
\ddot{w}_{1}+\omega_{1}^{2} w_{1}+a_{1} \dot{w}_{1}+a_{2} w_{1} \cos \Omega_{2} t+a_{3} w_{1}^{2}+a_{4} w_{2}^{2}+a_{5} w_{1} w_{2}^{2} \\
+a_{6} w_{1}^{3}+a_{7} w_{1} w_{2}=f_{1} \cos \Omega_{1} t \\
\ddot{w}_{2}+\omega_{2}^{2} w_{2}+b_{1} \dot{w}_{2}+b_{2} w_{2} \cos \Omega_{2} t+b_{3} w_{1} w_{2}+b_{4} w_{1}^{2}+b_{5} w_{2}^{2} \\
+b_{6} w_{2} w_{1}^{2}+b_{7} w_{2}^{3}=f_{2} \cos \Omega_{1} t
\end{array}
$$

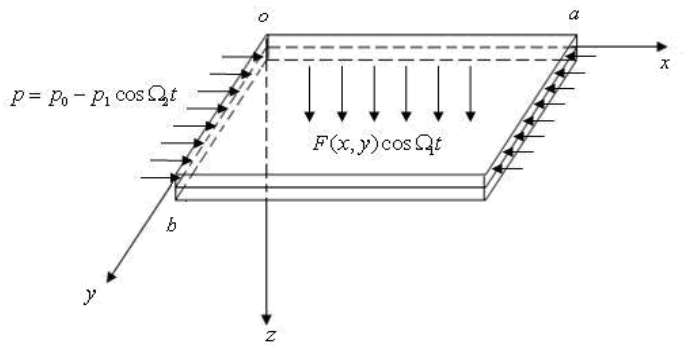

Fig. 1. The model of a FGM rectangular plate and the coordinate system.

where $\omega_{1}$ and $\omega_{2}$ are the amplitudes of two modes and all the coefficients can be found in paper [1]. Hao et al. [1] transformed the aforementioned equations into the averaged equations in subsequent form.

$$
\begin{gathered}
\dot{x}_{1}=\mu_{1} x_{1}+\left(\sigma_{1}+\alpha\right) x_{2}+\gamma_{1} x_{4}+N f_{1}^{1} \\
\dot{x}_{2}=\left(-\sigma_{1}+\alpha\right) x_{1}+\mu_{1} x_{2}+\gamma_{1} x_{3}+N f_{2}^{1} \\
\dot{x}_{3}=\gamma_{2} x_{2}+\mu_{2} x_{3}+\left(\sigma_{2}+\beta\right) x_{4}+N f_{3}^{1} \\
\dot{x}_{4}=\gamma_{2} x_{1}+\left(-\sigma_{2}+\beta\right) x_{3}+\mu_{2} x_{4}+N f_{4}^{1}
\end{gathered}
$$

where $\alpha=\alpha_{1}+\alpha_{2} f_{1}+\alpha_{11} f_{2}, \beta=\beta_{1}+\beta_{2} f_{1}+\beta_{3} f_{2}, \gamma_{1}=\alpha_{3} f_{2}+\alpha_{11} f_{1}, \gamma_{2}=\beta_{4} f_{2}+\beta_{5} f_{1}$ and you can find $\alpha_{i}(i=1,2,3,11)$ and $\beta_{i}(i=1, \cdots 5)$ in paper [1]. The nonlinear functions $N f_{i}^{1}(i=1, \ldots 4)$ can be found in [18]. Especially, $\mu_{i}(i=1,2)$ are the corresponding damping parameters. The resonant relations are $\omega_{1}=\frac{\Omega_{1}}{2}+\varepsilon^{2} \sigma_{1}, \omega_{2}=\frac{\Omega_{1}}{2}+\varepsilon^{2} \sigma_{2}, \Omega_{1}=\Omega_{2}=\Omega$, where $\sigma_{i}(i=1,2)$ are the two detuning parameters.

The Jacobi matrix of (1) evaluated at the initial equilibrium solution $\left(x_{1}, x_{2}, x_{3}, x_{4}\right)=(0,0,0,0)$ is as follows:

$$
J=\left[\begin{array}{cccc}
\mu_{1} & \sigma_{1}+\alpha & 0 & \gamma_{1} \\
-\sigma_{1}+\alpha & \mu_{1} & \gamma_{1} & 0 \\
0 & \gamma_{2} & \mu_{2} & \sigma_{2}+\beta \\
\gamma_{2} & 0 & -\sigma_{2}+\beta & \mu_{2}
\end{array}\right]
$$

we may obtain the characteristic polynomial of (2) 


$$
f(\lambda)=\lambda^{4}+b_{1} \lambda^{3}+b_{2} \lambda^{2}+b_{3} \lambda+b_{4}
$$

where

$$
\begin{gathered}
b_{1}=-2\left(\mu_{1}+\mu_{2}\right) \\
b_{2}=\sigma_{1}^{2}+\sigma_{2}^{2}+\mu_{1}^{2}+\mu_{2}^{2}-\alpha^{2}-\beta^{2}+4 \mu_{1} \mu_{2}-2 \gamma_{1} \gamma_{2} b_{3}=-2 \mu_{1} \mu_{2}^{2}-2 \mu_{2} \mu_{1}^{2}+2 \mu_{1} \gamma_{1} \gamma_{2}-2 \mu_{1} \sigma_{2}^{2}+2 \mu_{2} \gamma_{1} \gamma_{2} \\
-2 \sigma_{1}^{2} \mu_{2}+2 \mu_{1} \beta^{2}+2 \mu_{2} \alpha^{2} \\
b_{4}=-\mu_{1}^{2} \beta^{2}-\sigma_{1}^{2} \beta^{2}-2 \mu_{1} \mu_{2} \gamma_{1} \gamma_{2}+\gamma_{1}^{2} \gamma_{2}^{2}-\alpha^{2} \sigma_{2}^{2} \\
+\alpha^{2} \beta^{2}+\sigma_{1}^{2} \sigma_{2}^{2}-2 \sigma_{1} \sigma_{2} \gamma_{1} \gamma_{2}-2 \alpha \beta \gamma_{1} \gamma_{2}
\end{gathered}
$$

By Hurwitz criterion [20], we may obtain that when

$$
\begin{gathered}
b_{1}>0, b_{1} b_{2}-b_{3}>0, b_{4}>0, \\
b_{3}\left(b_{1} b_{2}-b_{3}\right)-b_{1}^{2} b_{4}>0
\end{gathered}
$$

the initial equilibrium solution $\left(x_{1}, x_{2}, x_{3}, x_{4}\right)=(0,0,0,0)$ is stable, otherwise the initial equilibrium solution is unstable, and bifurcation may occur. In the next section, the detailed analysis on the stability and bifurcation will be discussed when conditions (5) are not satisfied.

\section{Stability and bifurcation analysis}

\section{A double zero eigenvalues}

Taking parameters as $\mu_{1}=0, \mu_{2}=-2, \sigma_{1}=\sigma_{2}=1, \alpha=\frac{3}{4}, \beta=\frac{5}{4}, \gamma_{1}=\frac{1}{4}, \gamma_{2}=\frac{7}{4}$, $\alpha_{4}=\alpha_{5}=\alpha_{6}=\alpha_{7}=\alpha_{8}=\alpha_{9}=\alpha_{10}=1 \beta_{6}=\beta_{7}=\beta_{8}=\beta_{9}=\beta_{10}=\beta_{11}=1$.

Choosing $\sigma_{1}$ and $\sigma_{2}$ as perturbation parameters, using parameter transformation $\sigma_{1}=1+\delta_{1}$, $\sigma_{2}=1+\delta_{2}$, so the characteristic equation (3) becomes

$$
f(\lambda)=\lambda^{4}+b_{1} \lambda^{3}+b_{2} \lambda^{2}+b_{3} \lambda+b_{4}
$$

where,

$$
\begin{gathered}
b_{1}=4, \\
b_{2}=\delta_{1}^{2}+\delta_{2}^{2}+2 \delta_{1}+2 \delta_{2}+3, \\
b_{3}=4 \delta_{1}^{2}+8 \delta_{1}, \\
b_{4}=\delta_{1}^{2} \delta_{2}^{2}+2 \delta_{1}^{2} \delta_{2}+2 \delta_{2}^{2} \delta_{1}+\frac{7}{16} \delta_{2}^{2}+\frac{55}{16} \delta_{1}^{2} \\
+\frac{25}{8} \delta_{1} \delta_{2}+6 \delta_{1}
\end{gathered}
$$

We can see, $\Delta_{1}=b_{1}=4>0$, however, $\Delta_{3}=b_{3}\left(b_{1} b_{2}-b_{3}\right)-b_{1}^{2} b_{4}=-7\left(\delta_{1}-\delta_{2}\right)^{2} \leq 0$, therefore, the initial equilibrium is unstable.

\section{A simple zero and a pair of pure imaginary eigenvalues}

Taking parameters as

$\mu_{1}=-1, \quad \mu_{2}=\beta=\sigma_{1}=\gamma_{2}=0, \alpha=\sigma_{2}=\gamma_{1}=1, \quad \alpha_{4}=\alpha_{5}=\alpha_{6}=\alpha_{8}=\alpha_{9}=\alpha_{10}=-1 \quad, \quad \alpha_{7}=1$, $\beta_{6}=\beta_{7}=\beta_{9}=\beta_{10}=\beta_{11}=-1, \beta_{8}=1$.

Choosing $\sigma_{1}$ and $\sigma_{2}$ as perturbation parameters, using the parameter transformation $\sigma_{1}=\delta_{1}$, $\sigma_{2}=1+\delta_{2}$ and then introducing the subsequent state variable transformation into equation (1). 


$$
\left[\begin{array}{l}
x_{1} \\
x_{2} \\
x_{3} \\
x_{3}
\end{array}\right]=\left[\begin{array}{cccc}
1 & \frac{2}{5} & -\frac{1}{5} & 1 \\
1 & \frac{3}{5} & -\frac{4}{5} & -1 \\
0 & 1 & 0 & 0 \\
0 & 0 & 1 & 0
\end{array}\right]\left[\begin{array}{l}
z_{1} \\
z_{2} \\
z_{3} \\
z_{4}
\end{array}\right]
$$

Now equation (1) is transformed into a new system

$$
\begin{gathered}
\dot{z}_{1}=\frac{1}{10}\left(\delta_{1}-5 \delta_{2}\right) z_{2}-\frac{1}{10}\left(3 \delta_{1}+5 \delta_{2}\right) z_{3}-\delta_{1} z_{4}+N f_{1}^{2} \\
\dot{z}_{2}=z_{3}+\delta_{2} z_{3}+N f_{2}^{2} \\
\dot{z}_{3}=-z_{2}-\delta_{2} z_{2}+N f_{3}^{2} \\
\dot{z}_{4}=-2 z_{4}+\delta_{1} z_{1}+\frac{1}{10}\left(5 \delta_{1}+3 \delta_{2}\right) z_{2} \\
+\frac{1}{10}\left(\delta_{2}-5 \delta_{1}\right) z_{3}+N f_{4}^{2}
\end{gathered}
$$

where, the nonlinear functions $N f_{i}^{2}(i=1, \ldots, 4)$ are exhibited in the Appendix of paper [18].

Applying the systematic technique [12] to the equation (7), we obtain the normal form of (7) in cylindrical co-ordinates as follows:

$$
\begin{gathered}
\dot{y}=y\left(-\frac{6}{5} r^{2}-2 y^{2}\right) \\
\dot{r}=r\left(\frac{14}{25} r^{2}+\frac{14}{5} y^{2}\right)
\end{gathered}
$$

and

$$
\dot{\theta}=1+\delta_{2}-\frac{32}{25} r^{2}-\frac{12}{5} y^{2}
$$

It is easy to see that the steady state solution is only $y=r=0$. So the system (7) only has the initial equilibrium solution $\left(x_{1}, x_{2}, x_{3}, x_{4}\right)=(0,0,0,0)$.

\section{Two different pairs of pure imaginary eigenvalues (non-resonance)}

Taking parameters as $\mu_{1}=1, \mu_{2}=-1, \alpha=-\frac{1}{4}, \beta=\frac{1}{10}, \sigma_{1}=-\frac{1}{4}, \sigma_{2}=\frac{1}{10}, \gamma_{1}=5, \gamma_{2}=-\frac{1}{2}$, $\alpha_{5}=\frac{5}{3}, \alpha_{8}=\frac{8}{3}, \alpha_{10}=1, \alpha_{4}=\alpha_{6}=\alpha_{7}=\alpha_{9}=0, \beta_{6}=\beta_{7}=\beta_{8}=\beta_{9}=\beta_{10}=\beta_{11}=0$.

Choosing $\sigma_{1}$ and $\sigma_{2}$ as perturbation parameters, using the parameter transformation $\sigma_{1}=-\frac{1}{4}+\delta_{1}$, $\sigma_{2}=\frac{1}{10}+\delta_{2}$. Then, introducing the state variable transformation

$$
\left[\begin{array}{l}
x_{1} \\
x_{2} \\
x_{3} \\
x_{4}
\end{array}\right]=\left(\begin{array}{cccc}
-2 & -2 & -2 & -2 \sqrt{2} \\
2 & 0 & -2 & 0 \\
-\frac{2}{5} & \frac{2}{5} & \frac{2}{5} & -\frac{2}{5} \sqrt{2} \\
1 & 0 & 1 & 0
\end{array}\right)\left[\begin{array}{l}
z_{1} \\
z_{2} \\
z_{3} \\
z_{4}
\end{array}\right]
$$


into (1) yields

$$
\begin{gathered}
\dot{z}_{1}=z_{2}+\left(\frac{1}{2} \delta_{1}+\frac{1}{5} \delta_{2}\right) z_{1}+\left(\frac{1}{2} \delta_{1}-\frac{1}{5} \delta_{2}\right) z_{2}+\left(\frac{1}{2} \delta_{1}-\frac{1}{5} \delta_{2}\right) z_{3}+\sqrt{2}\left(\frac{1}{2} \delta_{1}+\frac{1}{5} \delta_{2}\right) z_{4}+N f_{1}^{3}(11 \mathrm{a}) \\
\dot{z}_{2}=-z_{1}+\frac{21}{20} \delta_{2} z_{1}+\left(\frac{1}{2} \delta_{1}+\frac{1}{5} \delta_{2}\right) z_{2}+\left(\frac{29}{20} \delta_{2}+\delta_{1}\right) z_{3}+\sqrt{2}\left(\frac{1}{2} \delta_{1}-\frac{1}{5} \delta_{2}\right) z_{4}+N f_{2}^{3} \\
\dot{z}_{3}=\sqrt{2} z_{4}+\left(\frac{1}{5} \delta_{2}-\frac{1}{2} \delta_{1}\right) z_{1}-\left(\frac{1}{2} \delta_{1}+\frac{1}{5} \delta_{2}\right) z_{2} \\
-\left(\frac{1}{2} \delta_{1}+\frac{1}{5} \delta_{2}\right) z_{3}-\sqrt{2}\left(\frac{1}{2} \delta_{1}-\frac{1}{5} \delta_{2}\right) z_{4}+N f_{3}^{3}(11 \mathrm{c}) \dot{z}_{4}=-\sqrt{2} z_{3}-\frac{\sqrt{2}}{2}\left(\delta_{1}+\frac{29}{20} \delta_{2}\right) z_{1}-\frac{\sqrt{2}}{2}\left(\frac{1}{2} \delta_{1}-\frac{1}{5} \delta_{2}\right) z_{2} \\
-\frac{21}{40} \sqrt{2} \delta_{2} z_{3}-\left(\frac{1}{2} \delta_{1}+\frac{1}{5} \delta_{2}\right) z_{4}+N f_{4}^{3}
\end{gathered}
$$

where, the nonlinear functions $N f_{i}^{3}(i=1, \ldots, 4)$ are exhibited in the Appendix of paper [18]. With the same method [13], we get the normal form in polar coordinate system as follows:

$$
\begin{aligned}
& \dot{r}_{1}=r_{1}\left(\frac{1}{2} \delta_{1}+\frac{1}{5} \delta_{2}-\frac{3}{25} r_{1}^{2}+\frac{28}{75} r_{2}^{2}\right) \\
& \dot{r}_{2}=r_{2}\left(-\frac{1}{2} \delta_{1}-\frac{1}{5} \delta_{2}-\frac{4}{25} r_{1}^{2}+\frac{1}{75} r_{2}^{2}\right)
\end{aligned}
$$

and

$$
\begin{aligned}
& \dot{\theta}_{1}=1+\frac{1}{4} \delta_{1}-\frac{5}{8} \delta_{2}+\frac{109}{120} r_{1}^{2}+\frac{107}{300} r_{2}^{2} \\
& \dot{\theta}_{2}=\sqrt{2}-\frac{\sqrt{2}}{4} \delta_{1}+\frac{29}{80} \sqrt{2} \delta_{2}+\sqrt{2}\left(-\frac{41}{150} r_{1}^{2}-\frac{299}{600} r_{2}^{2}\right)
\end{aligned}
$$

On the basis of (12), the steady state solutions are obtained by setting $\dot{r}_{1}=\dot{r}_{2}=0$, which fall into four categories and the Jacobi matrix of (12) is as follows:

$$
J=\left[\begin{array}{cc}
\frac{1}{2} \delta_{1}+\frac{1}{5} \delta_{2}-\frac{9}{25} r_{1}^{2}+\frac{28}{75} r_{2}^{2} & \frac{56}{75} r_{1} r_{2} \\
-\frac{8}{25} r_{1} r_{2} & -\frac{1}{2} \delta_{1}-\frac{1}{5} \delta_{2}-\frac{4}{25} r_{1}^{2}+\frac{3}{75} r_{2}^{2}
\end{array}\right]
$$

(1).The initial equilibrium solution (E.S.):

$$
r_{1}=r_{2}=0
$$

The stability conditions for the E.S. which can be obtained by evaluating the Jacobi matrix (14) at the initial equilibrium solution are $5 \delta_{1}+2 \delta_{2}<0$ and $5 \delta_{1}+2 \delta_{2}>0$, therefore the initial equilibrium solution is unstable.

(2). The incipient Hopf bifurcation solution (H.B. ( I ) with frequency $\omega_{1}$ ):

$$
\begin{aligned}
& r_{1}^{2}=\frac{5}{6}\left(5 \delta_{1}+2 \delta_{2}\right), r_{2}=0 \\
& \omega_{1}=1+\frac{581}{144} \delta_{1}+\frac{8}{9} \delta_{2}
\end{aligned}
$$

From (16), we can see that there exists H.B.( I ) solution when $5 \delta_{1}+2 \delta_{2}>0$. The stability conditions for the H.B.( I ) solution which can be obtained by evaluating the Jacobi matrix (14) at the H.B.( I ) solution are $5 \delta_{1}+2 \delta_{2}>0$. So there exists stable H.B. ( I ) solution when $5 \delta_{1}+2 \delta_{2}>0$.

(3). The secondary Hopf bifurcation solution (H. B. ( II ) with frequency $\omega_{2}$ ): 


$$
\begin{gathered}
r_{1}=0, r_{2}^{2}=\frac{15}{2}\left(5 \delta_{1}+2 \delta_{2}\right) \\
\omega_{2}=\sqrt{2}\left(1-\frac{303}{16} \delta_{1}-\frac{569}{80} \delta_{2}\right)
\end{gathered}
$$

From(17), we can see that there exists H.B.( II ) solution when $5 \delta_{1}+2 \delta_{2}>0$, But the stability conditions for the H.B.( II ) solution which are obtained by evaluating the Jacobi matrix (14) at the H.B.( II ) solution are $5 \delta_{1}+2 \delta_{2}<0$. So the H.B.( II ) solution is unstable in its existence region.

(4).Quasi-periodic solution (2-D torus with frequency $\left.\omega_{1}, \omega_{2}\right)$ :

$$
\begin{gathered}
r_{1}^{2}=-\frac{145}{218}\left(5 \delta_{1}+2 \delta_{2}\right), \\
r_{2}^{2}=-\frac{105}{218}\left(5 \delta_{1}+2 \delta_{2}\right) \\
\omega_{1}=1-\frac{18991}{5232} \delta_{1}-\frac{14237}{6540} \delta_{2} \\
\omega_{2}=\sqrt{2}\left(1+\frac{9727}{5232} \delta_{1}+\frac{31553}{26160} \delta_{2}\right)
\end{gathered}
$$

From (18), we can see that there exists quasi-periodic solution when $5 \delta_{1}+2 \delta_{2}<0$. The stability conditions can be determined by evaluating the trace and determinant of the Jacobi matrix (14) at quasi-periodic solution as follows,

$$
\begin{gathered}
\operatorname{Tr}=\frac{16}{109}\left(5 \delta_{1}+2 \delta_{2}\right)<0 \\
\operatorname{Det}=\frac{436}{1875} r_{1}^{2} r_{2}^{2}>0
\end{gathered}
$$

It is easy to see that (20) is automatically satisfied as long as the quasi-periodic solution exists. Therefore there exists stable quasi-periodic solution when $5 \delta_{1}+2 \delta_{2}<0$.

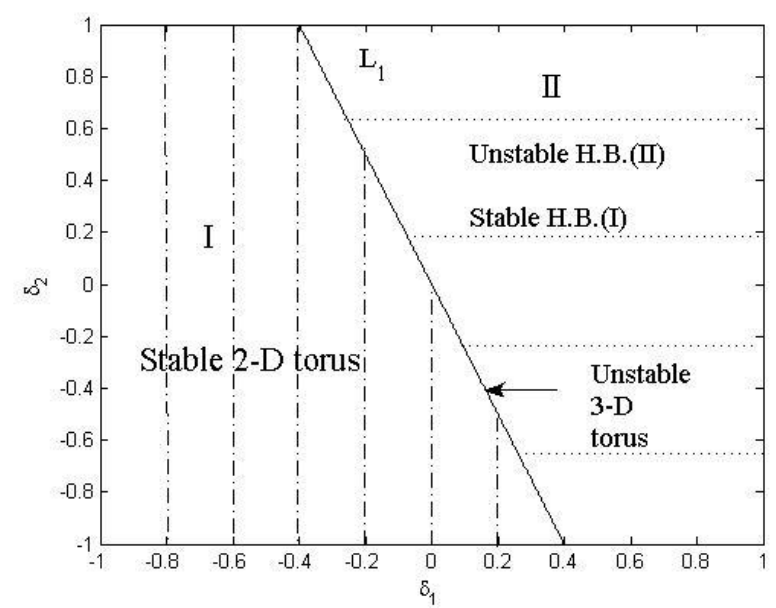

Fig. 2. Bifurcation diagram on detuning parameters for the case of two different pairs of pure imaginary eigenvalues 

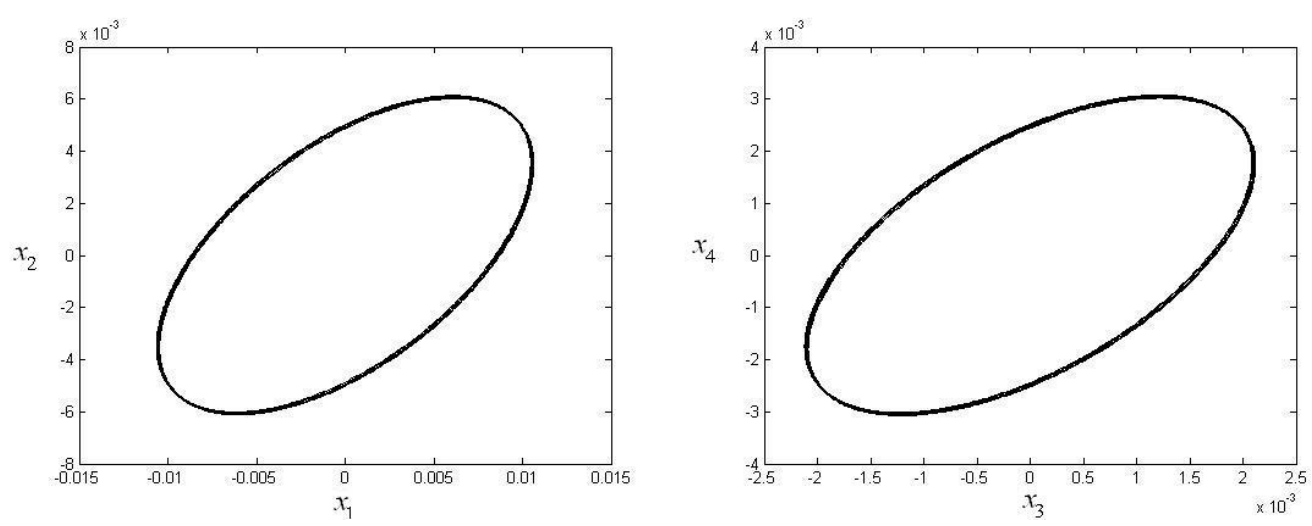

Fig. 3. Trajectory projection of H.B. (1) solution starting from $\left(x_{1}, x_{2}, x_{3}, x_{4}\right)=(0.0020,-0.0042,0.0021,0.0021)$ when $\left(\delta_{1}, \delta_{2}\right)=(0.001,-0.002)$
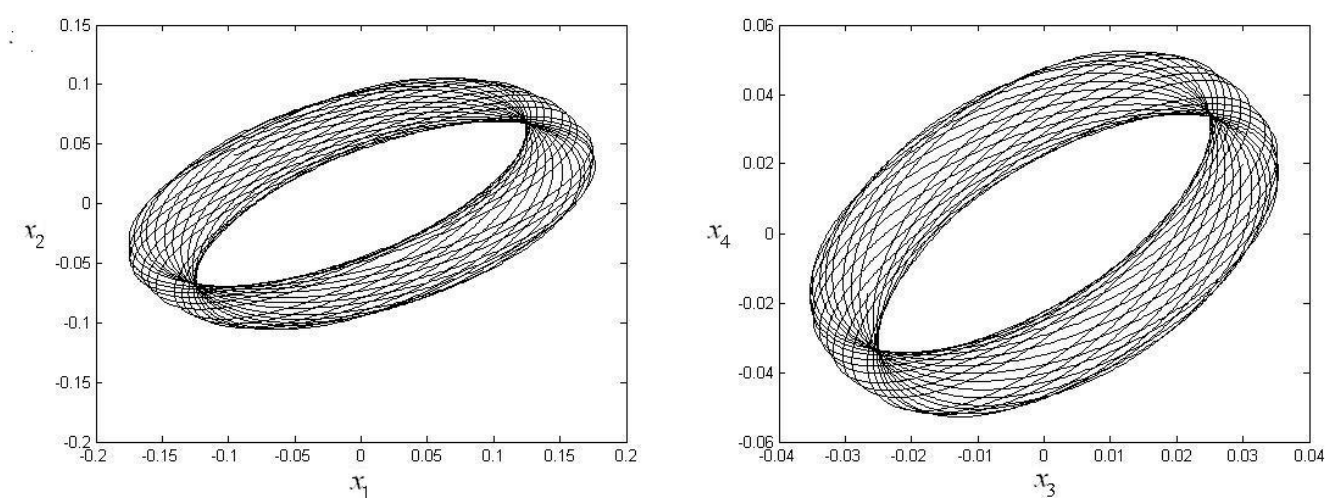

Fig. 4. A two-dimensional torus starting from $\left(x_{1}, x_{2}, x_{3}, x_{4}\right)=(0.06,-0.02,0.03,0.02)$ when $\left(\delta_{1}, \delta_{2}\right)=(-0.0001,-0.0002)$

From the previous analysis, we can get a critical line $L_{1}: 5 \delta_{1}+2 \delta_{2}=0$, from which the stable quasiperiodic solution loses its stability and bifurcates into an unstable 3-D torus [13] as shown in Fig.2. When $\left(\delta_{1}, \delta_{2}\right)$ in region II, there exists unstable secondary Hopf bifurcation solution.

Now choosing a point $\left(\delta_{1}, \delta_{2}\right)=(0.001,-0.002)$, which is located in the stable region for H.B.( I ) solution, one can find a Hopf bifurcation solution as shown in Fig.3., where the initial point is chosen as $\left(x_{1}, x_{2}, x_{3}, x_{4}\right)=(0.0020,-0.0042,0.0021,0.0021)$ When $\left(\delta_{1}, \delta_{2}\right)=(-0.0001,-0.0002)$, a $2-\mathrm{D}$ torus exists where the initial point is chosen as $\left(x_{1}, x_{2}, x_{3}, x_{4}\right)=(0.06,-0.02,0.03,0.02)$ as shown in Fig.4.

\section{Conclusions}

In this paper, the functionally graded material (FGM) rectangular plate on the condition of detuning parameters as perturbation parameters has been studied in detail with both analytical and numerical approaches. With the aid of normal form theory and symbolic computation language Maple, we simplify the original set of differential equations and get steady state solutions as well as their stability conditions respectively. With fourth-order Runge-Kutta method, the bifurcation diagrams and trajectory projections are obtained all numerical solutions agree with the analytical predictions qualitatively.

\section{Acknowledgements}

The authors greatly appreciate the anonymous reviews for their insightful comments and suggestions 
for further improving the quality of this work. This work was supported by the Natural Science Foundation of the Jiangsu Higher Education Institutions of China (No.16KJD110007).

\section{References}

[1] Y. X. Hao, L.H. Chen, W. Zhang, J.G .Lei, Nonlinear oscillations, bifurcations and chaos of functionally graded materials plate, J. Sound Vib.312 (2008) 862-892.

[2] J. Yang, H.S. Shen, Vibration characteristics and transient response of shear deformable functionally graded plates in thermal environment, J. Sound Vib. 255 (2002) 579-602.

[3] X.L. Huang, H.S. Shen, Nonlinear vibration and dynamic response of functionally graded plates in thermal environments, International journal of solids and structures 41(2004)2403-2427.

[4] J. Yang, H.S. Shen, Free vibration and parametric resonance of shear deformable functionally graded cylindrical panels, J. Sound Vib. 216 (2003) 871-893.

[5] Y.D. Hu, Z.Q. Zhang, Bifurcation and chaos of thin circular functionally graded plate in thermal environment, Chaos, Solitons and Fractals 44 (2011) 739-750.

[6] M.T. Piovan, R. Sampaio, Vibrations of axially moving flexible beams made of functionally graded materials, Thin-Walled Structures 46 (2008) 112-121.

[7] P. Yu, K. Huseyin, Static and dynamic bifurcations associated with a double-zero eigenvalue, Dynamics and Stability of Systems 1 (1986) 73-86.

[8] P. Yu, K. Huseyin, A perturbation analysis of interactive static and dynamic bifurcations, IEEE transactions on automatic control, 33(1988) 28-41.

[9] P. Yu, K. Huseyin. On bifurcation into nonresonant quasi-periodic motions, Appl. Math. Modelling 12 (1988) 189-201.

[10] P. Yu, K. Huseyin, Bifurcations associated with a three-fold zero eigenvalue, Quarterly of applied mathematics, 46(2) (1988) 193-216.

[11] P. Yu, Huseyin K. Bifurcations associated with a double zero and a pair of pure imaginary eigenvalues. SIAM Journal of Applied Mathematics, 48(2) 1988 229-261.

[12] Q. Bi, P. Yu, Symbolic computation of normal forms for semi-simple cases, Journal of Computational and Applied Mathematics, 102 (1999) 195-220.

[13] P. Yu, Analysis on double Hopf bifurcation using computer algebra with the aid of multiple scales, Nonl. Dyn. 27(2002) 19-53.

[14] P. Yu, Computation of norm forms via a perturbation technique, J. Sound Vib. 211(1998) 19-38.

[15] P. Yu, Symbolic computation of normal forms for resonant double Hopf bifurcations using a perturbations using a perturbation technique, J. Sound Vib. 247 (2001) 615-632.

[16] P. Yu, Q. Bi, Analysis of non-linear dynamics and bifurcations of a double pendulum, J. Sound Vib. 217 (1998) 691-736.

[17] P.Yu, W. Zhang, Q. Bi, Vibration analysis on a thin plate with the aid of computation of normal forms, Int. J. Non-linear Mech. 36 (2001) 597-627.

[18] X.H. Zhang, F.Q. Chen, H.L. Zhang, Stability and local bifurcation analysis of functionally graded material plate under transversal and in-plane excitations, Appl. Math. Modelling 37 (2013) 6639-6651.

[19] K. Ogata, Modern control engineering, Englewood Cliffs, NJ: Prentice-Hall, (1970) 